\title{
Estudo fitoquímico da espécie Pterocaulon interruptum DC. (Asteraceae)
}

\author{
Ana Carolina Winkler Heemann', Obdúlio Gomes Miguel ${ }^{1 *}$, Marilis Dallarmi Miguel', Cristina \\ Mayumi Sasaki', Franco Delle Monache ${ }^{2}$
}

${ }^{1}$ Departamento de Farmácia, Universidade Federal do Paraná, ${ }^{2}$ Universidade Católica de Roma

*Correspondência:

O. G. Miguel

Departamento de Farmácia

Universidade Federal do Paraná

Av. Prefeito Lothário Meissner, 632 -

Jardim Botânico

80210-170 - Curitiba - PR, Brasil

E-mail: obdulio@ufpr.br
A análise fitoquímica das partes aéreas de Pterocaulon interruptum DC. (Asteraceae) resultou no isolamento de cinco compostos: uma cumarina, sabandinol, dois esteróides, estigmasterol e 3-O-acetiltaraxasterol e dois flavonóides, quercetina e taxifolina 7-Oprenilada. As estruturas destas substâncias foram estabelecidas por análises espectroscópicas, sendo que este é o primeiro trabalho sobre $o$ isolamento destes compostos em Pterocaulon interruptum $D C$.

\section{INTRODUÇÃO}

O gênero Pterocaulon, compreendendo cerca de 30 espécies (Missouri Botanical Garden, 2001), caracterizase por espécies perenes, de caules eretos com folhas lanceoladas e lanosas apresentando tricomas glandulares e tectores (Cabrera, 1963). Apresentam distribuição bicêntrica, estendendo-se do sul dos Estados Unidos até a Argentina e em países Asiáticos, não ocorrendo na Europa e África (Cabrera, Ragonese, 1978). De acordo com Angely (1965), estudos sobre a flora do estado do Paraná revelaram a presença de quatro espécies do gênero Pterocaulon: P. alopecuroideum (Lamarck) DC., P. angustifolium DC., P. interruptum DC. e P. rugosum (Vahl.) Malme. Algumas cumarinas isoladas de espécies de Pterocaulon podem ser citadas: 6,7-dimetoxicumarina de $P$. sphacelatum, oito cumarinas do extrato etéreo das partes aéreas de $P$. balansae e P. lanatum (MAGALHÃES et al., 1981) e uma cumarina 5,6,7,8tetraoxigenada, denominada purpurenol de $P$. purpurascens (Debenedetti et al., 1991). Debenedetti et al. (1997) realizaram uma revisão estrutural das cumarinas denominadas sabandinol e sabandinona e a descrição de mais duas novas cumarinas denominadas de virgatenol e virgatol (Debenedetti et al., 1998). Também foram isoladas de $P$. virgatum as seguintes substâncias: taraxasterol, esqualeno, taxifolina 7-O-prenilada, entre outros compostos (Bohlmann et al., 1981). As partes aéreas de $P$. purpurascens são utilizadas na medicina popular argentina devido a suas propriedades digestivas e as partes aéreas de $P$. polystachium como repelente de pulgas e moscas (Debenedetti et al., 1994). As espécies do gênero Pterocaulon apresentam cumarinas, conhecidas como marcadores quimiotaxonômicos da família Asteraceae (Silva, 1978). Salienta-se a importância da quimiotaxonomia na área farmacêutica, uma vez que aliada a estudos etnofarmacológicos são aumentadas as possibilidades de se desenvolver novos fármacos.

A relevância do presente estudo caracteriza-se pelas inúmeras propriedades biológicas descritas para outras espécies de Pterocaulon. A espécie Pterocaulon virgatum (L.) DC. é descrita como digestiva (Martino et al., 1979), a espécie Pterocaulon sphacelatum foi efetiva no tratamento de infecções respiratórias, irritações oculares e também como inibidora na replicação de poliovírus tipo 1 (Semple et al., 1998). Justifica-se, também, a realização deste estudo como forma de contribuição ao estudo da espécie Pterocaulon interruptum DC., para a qual não há descri- 
ção em estudos anteriores. (Heemann, 2002; Heemann, Miguel, Miguel, 2004).

\section{MATERIAL E MÉTODOS}

A espécie Pterocaulon interruptum DC. foi coletada nos meses de janeiro e fevereiro de 2001 no litoral de Santa Catarina, Município de Piçarras. A identificação da espécie vegetal foi realizada pelo Botânico Dr. G. Hatschbach, do Museu Botânico Municipal de CuritibaPR, e a exsicata foi depositada no herbário deste museu, registrada sob número 266.085 .

As partes aéreas de Pterocaulon interruptum (420 g) secas e estabilizadas em estufa com circulação de ar foram extraídas em Soxhlet, com solventes de polaridade crescente, hexano, clorofórmio, acetato de etila e metanol, utilizando o resíduo da primeira extração para retomar a próxima extração, obtendo-se como rendimento de 19,06 g (4,3\%), $6,7 \mathrm{~g}(1,59 \%), 3,6 \mathrm{~g}(0,85 \%)$ e $19,7 \mathrm{~g}(4,86 \%)$, respectivamente. Após cada extração, as frações obtidas foram concentradas no próprio Soxhlet, ao qual foi adaptada uma saída lateral.

As quatro frações foram processadas da mesma forma, previamente dissolvidas em metanol e, em seguida, incorporadas a 6 g de sílica-gel 60 para formação da pastilha. Posteriormente, foram submetidas à cromatografia em coluna $(30 \times 3 \mathrm{~cm})$ empacotada com $30 \mathrm{~g}$ sílica-gel 60 . A fração incorporada na forma de pastilha foi eluída com sistema de solventes de polaridade crescente, sendo utilizado hexano, acetato de etila e metanol. O controle da eluição foi realizado com lâmpada ultravioleta a $360 \mathrm{~nm}$. As frações foram analisadas por cromatografia em camada delgada revelada com luz UV e as frações semelhantes foram reunidas.

Os espectros de massas foram obtidos em espectrômetro Shimadzu CHMS-Qp 2000 A e os espectros no infravermelho em equipamento Bomen - Hartmann \& Braum MB-Série, em pastilha comprimida de brometo de potássio anidro. Os espectros de $\mathrm{RMN}$ de ${ }^{13} \mathrm{C}$ e de ${ }^{1} \mathrm{H}$ foram obtidos em equipamento Varian XL 300 a $75 \mathrm{MHz}$ (Universidade Católica de Roma, Itália).

\section{RESULTADOS E DISCUSSÃO}

A investigação fitoquímica das partes aéreas de Pterocaulon interruptum DC. (Asteraceae) resultou no isolamento da cumarina sabandinol (1), dos triterpenos estigmasterol (2) e 3-O-acetil taraxasterol (3) e dos flavonóides quercetina (4), taxifolina 7-O-prenilada (5). Da fração hexânica isolou-se e caracterizou-se uma cumarina 5,6,7,8-tetraoxigenada denominada sabandinol (300 mg).
Da fração acetato de etila foram isolados e caracterizados os esteróides estigmasterol $(8,4 \mathrm{mg})$ e 3-O-acetil taraxasterol $(70,7 \mathrm{mg})$. Da fração clorofórmica os flavonóides quercetina $(8,9 \mathrm{mg})$ e taxifolina $7-O$-prenilada $(23,9 \mathrm{mg})$. Não foram isoladas substâncias da fração metanólica .

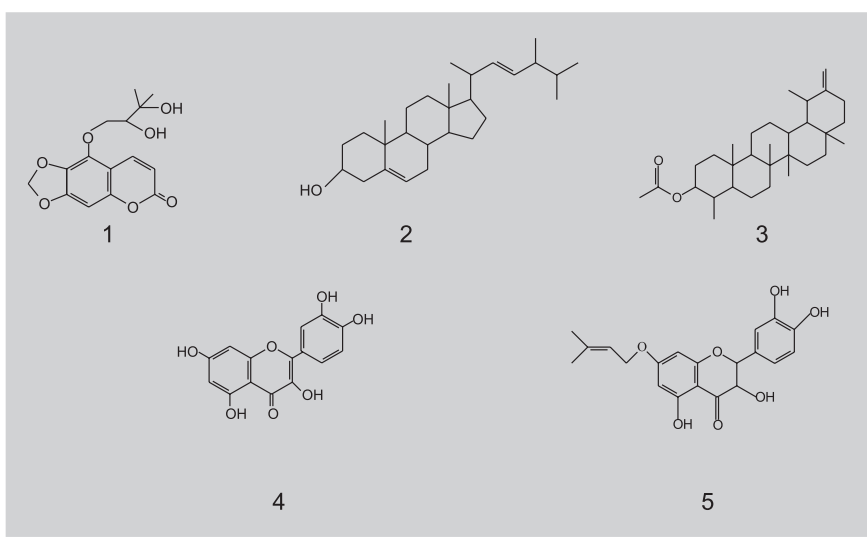

FIGURA 1. Substâncias isoladas e caracterizadas de Pterocaulon interruptum DC. (Asteraceae).

As estruturas destas substâncias foram elucidadas a partir de dados obtidos por análises espectroscópicas de RMN de ${ }^{13} \mathrm{Ce}$ de ${ }^{1} \mathrm{H}, \mathrm{IV}, \mathrm{UV}$ e EM e por comparação com dados da literatura.

A cumarina 1 apresentou bandas em $3400 \mathrm{~cm}^{-1}(\mathrm{OH})$ e $1700 \mathrm{~cm}^{-1}$ (carbonila) quando realizado o espectro no IV. O UV apresentou máximos de absorção em 316 e $235 \mathrm{~nm}$. O espectro de massas apresentou os seguintes picos principais: $m / z 308$ (100), $\mathrm{m} / z$ 206, $\mathrm{m} / z$ 178, $\mathrm{m} / \mathrm{z} 59$ e $\mathrm{m} / \mathrm{z} 43$. Os dados de $\mathrm{RMN} \mathrm{de}{ }^{13} \mathrm{C} \mathrm{e}{ }^{1} \mathrm{H}$ obtidos experimentalmente corroboram a literatura (Debenedetti et al., 1997).

Os esteróides 2 e 3 apresentaram sinais em $\mathrm{RMN}{ }^{13} \mathrm{C}$ que os caracterizam de acordo com comparação dos dados registrados na literatura (Debenedetti et al., 1994). Realizado o espectro no IV, os esteróides 2 e 3 apresentaram banda larga em $3400 \mathrm{~cm}^{-1}(\mathrm{OH})$ e bandas em 2959, 2935 e $2836 \mathrm{~cm}^{-1}$, normais de metilas e metilenos alifáticos, sendo que o triterpeno 3 apresentou, ainda, uma banda em $1724 \mathrm{~cm}^{-1}$, caracterizando a carbonila do grupamento acetila, presente na posição 3 .

Os flavonóides 4 e 5 apresentaram em IV, banda larga em $3409 \mathrm{~cm}^{-1}(\mathrm{OH})$, em $1663 \mathrm{~cm}^{-1}$, indicando a presença de carbonila conjugada e, ainda, em $1611 \mathrm{~cm}^{-1}$ característica de insaturações.

O flavonóide 4, no espectro de massas, apresentou $\mathrm{m} / \mathrm{z} 302$ (100) e outros íons fragmentários $\mathrm{m} / \mathrm{z} 273, \mathrm{~m} / \mathrm{z}$ $257, \mathrm{~m} / z 229, \mathrm{~m} / \mathrm{z} 153, \mathrm{~m} / \mathrm{z} 137$ e $\mathrm{m} / \mathrm{z} 109$, típicos de estruturas flavonoídicas. O espectro no UV apresentou máximos 
de absorção em $371 \mathrm{~nm}$ e $255 \mathrm{~nm}$ e uma banda com baixo coeficiente de extinção em 304 nm, correspondendo a absorções típicas de flavonóis (Mabry, Markham, Thomas, 1970). Os espectros obtidos de RMN de ${ }^{13} \mathrm{C} \mathrm{e}{ }^{1} \mathrm{H}$ contribuíram para a caracterização da substância como sendo a quercetina (Silva, Carvalho, Braz-Filho, 2003).

O flavonóide 5, no UV, apresentou máximo de absorção em $290 \mathrm{~nm}$ e uma banda com baixo coeficiente de extinção em $325 \mathrm{~nm}$, sendo que também foram realizados ensaios para confirmação das posições das hidroxilas. Dados de RMN de ${ }^{13} \mathrm{C}$ a $75 \mathrm{MHz}$, em acetona, e espectros em HETCOR permitiram caracterizar a substância como sendo a taxifolina 7-O-prenilada (Mabry, Markham, Thomas, 1970).

Para auxiliar na elucidação estrutural foram realizados ensaios no UV, os quais por meio de deslocamentos batocrômicos e hipsocrômicos em reação com diferentes substâncias como $\mathrm{NaOMe}, \mathrm{NaOAc}, \mathrm{H}_{3} \mathrm{BO}_{3}$ e $\mathrm{HCl}$ permitem detectar a presença de hidroxilas em diferentes posições das cadeias flavonoídicas (Mabry, Markham, Thomas, 1970).

\section{CONCLUSÕES}

Diversas atividades biológicas foram descritas em outras espécies do gênero Pterocaulon, o que suscitou o interesse no estudo desta espécie. A partir da análise fitoquímica da espécie Pterocaulon interruptum DC. (Asteraceae) foram isoladas e caracterizadas cinco substâncias já conhecidas, porém referenciadas pela primeira vez nesta espécie. A partir das substâncias isoladas é possível o planejamento da linha de estudos biológicos a serem realizados para a comprovação das atividades.

\section{ABSTRACT}

\section{Phytochemical study of Pterocaulon interruptum Dc., Asteraceae}

Chemical investigation of the aerial parts of Pterocaulon interruptum DC., Asteraceae, resulted in the isolation of five compounds: one coumarin, sabandinol, two steroids, stigmasterol and 3-O-acetyl taraxasterol and two flavonoids, quercetin (flavonol) and 7-O-prenyl taxifolin (dihydroxyflavonol). The structures of these compounds were established by spectroscopic analysis. This paper deals with the first report of these compounds in Pterocaulon interruptum $D C$.

UNITERMS: Coumarins. Triterpenes. Flavonoids.

\section{AGRADECIMENTOS}

Os autores agradecem ao botânico Dr. G. Hatschbach e ao biólogo O. dos S. Ribas do Museu Botânico Municipal pela identificação e depósito da espécie vegetal.

\section{REFERÊNCIAS BIBLIOGRÁFICAS}

ANGELY, J. Flora analítica do Paraná. São Paulo: Universidade de São Paulo, 1965. 728 p.

BOHLMANN, F.; ABRAHAM, W. R.; KING, R. M.; ROBINSON, H. Thiophene acetylenes and flavonols from Pterocaulon virgatum. Phytochemistry, Oxford, v. 20, n.4, p.825-827, 1981.

CABRERA, A.L. Flora de la provincia de Buenos Aires. Colección Científica del I.N.T.A., Buenos Aires, v. 4, n. $6,1963$.

CABRERA, A.L.; RAGONESE, A.M. Revisión del gênero Pterocaulon (Compositae). Darwiniana, Buenos Aires, v. 21, n.24, 1978.

DEBENEDETTI, S.L.; KIMPE, N.; BOEYKENS, M.; COUSSIO, J. D.; KESTELEYN, B. Structural revision of four coumarins from Pterocaulon species. Phytochemistry, Oxford, v.45, n.7, p.1515-1517, 1997.

DEBENEDETTI, S.L.; KIMPE, N.; BOEYKENS, M.; COUSSIO, J. D.; KESTELEYN, B. Polyphenols of Pterocaulon polystachium. Fitoterapia, Milano, v. 45, n.2, p. 188, 1994.

DEBENEDETTI, S. L.; NADINIC, E. L.; COUSSIO, J. D.; KIMPE, N. de; DUPON, J. F.; DECLERCQ, J. P. Purpurenol, a highly oxygenated coumarin from Pterocaulon purpurascens. Phytochemistry, Oxford, v. 30, n.8, p.2757-2758, 1991.

DEBENEDETTI, S. L.; NADINIC, E. L.; COUSSIO, J. D.; KIMPE, N.; BOEYKENS, M. Two 6,7-dioxygenated coumarins from Pterocaulon virgatum. Phytochemistry, Oxford, v. 48, n.4, p.707-710, 1998.

HEEMANN, A.C.W. Estudo fitoquímico, botânico e das propriedades antimicrobianas de Pterocaulon interruptum DC. (Asteraceae). Curitiba, 2002. $104 \mathrm{p}$. [Dissertação de Mestrado. Departamento de Farmácia. Universidade Federal do Paraná]. 
HEEMANN, A.C.W.; MIGUEL, O.G.; MIGUEL, M.D. Revisão do gênero Pterocaulon: aspectos fitoquímicos e atividades biológicas. Visão Acadêmica, Curitiba, v. 5, n.1, p. 53-60, 2004.

MABRY, T.J.; MARKHAM, K.R; THOMAS, M.B. The systematic identification of flavonoids. Berlin: Springer, 1970. $354 \mathrm{p}$.

MAGALHÃES, A. F.; MAGALHÃES, E. G.; LEITÃO FILHO, H. F.; FRIGHETTO, R. T. S.; BARROS, S. M. G. Coumarins from Pterocaulon balansae and Pterocaulon lanatum. Phytochemistry, Oxford, v.20, n.6, p.1369-1371, 1981

MARTINO, V.S.; DEBEBEDETTI, S.L.; COUSSIO, J.D. Caffeoylquinic acids from Pterocaulon virgatum and Pluchea sagittalis. Phytochemistry, Oxford, v. 18, p. 2052, 1979.
MISSOURI BOTANICAL GARDEN. Nomenclatural Data Base. Disponível em: http://mobot.mobot.org/cgi-bin/ search_vast $>$. Acesso em: 21 out. 2001.

SEMPLE, S.J.; REYNOLDS, G.D.; O'LEARY, M.C.; FLOWER, R.L.P. Screening of Australian medicinal plants for antiviral activity. J. Ethnopharmacol., London, v. 60, n.2, p.163-172, 1998.

SILVA, M.F.G.F. Métodos quimiossistemáticos: aplicação a famílias vegetais caracterizadas por cumarinas. São Paulo, 1978. 373p. [Tese de Doutorado. Instituto de Química. Universidade de São Paulo].

SILVA, T. M. S.; CARVALHO, M. G.; BRAZ-FILHO, R.; AGRA, M. F. Ocorrência de flavonas, flavonóis e seus glicosídeos em espécies do gênero Solanum (Solanaceae). Quím. Nova, São Paulo, v.26, n.4, p.517-522, 2003.

Recebido para publicação em 25 de maio de 2006 Aceito para publicação em 10 de outubro de 2006 